\title{
Optical beam tracking and distortion compensation based on a nonlinear lens mechanism
}

\author{
Alexander S. Koujelev \\ Alexander E. Dudelzak, MEMBER SPIE \\ Canadian Space Agency \\ 6767 Route de l'Aéroport \\ Longueuil, Québec, Canada J3Y 8Y9 \\ E-mail: alexander.koujelev@space.gc.ca
}

\begin{abstract}
Optical free-space communications involving moving parties require precise beam pointing and mutual tracking of communicating transceivers. The existing variety of tracking techniques is still the major limiting factor in free-space laser communications. Here we propose a technique for optical beam tracking that utilizes nonlinear optical properties of materials. In our proof-of-concept experiment, a thin layer of a nematic liquid crystal (NLC) with high thermal nonlinearity was used to produce a thermal lens induced by the incoming optical beam. That beam modulated the NLC refractive index. As the transmitted optical beam passed through the same layer, the beam intensity was modulated in the far field. A sharp intensity maximum was formed at the distant communicating party position. This tracking capability has been demonstrated for angular disturbances at a subkilohertz frequency. This tracking mechanism also offers adaptive capability of compensation of strong aberrations. Such compensation has been demonstrated experimentally; numerical modeling performed with the Fresnel integral technique showed very good agreement with the experiment. (๑) 2008 Goverment of Canada. [DOI: 10.1117/1.2968236]
\end{abstract}

Subject terms: free-space laser communications; optical tracking; wavefront distortion compensation; nonlinear optical devices; self-focusing; liquid crystals.

Paper 070967RR received Dec. 7, 2007; revised manuscript received May 20, 2008; accepted for publication May 23, 2008; published online Aug. 25, 2008. This paper is a revision of a paper presented at the SPIE conference on Atmospheric Optics: Models, Measurements, and Talget-in-the-Loop Propagation, August 2007, San Diego, California. The paper presented there appears (unrefereed) in SPIE Proceedings Vol. 6708.

\section{Introduction}

Optical free-space communication technology has become an attractive addition to microwave links, due to numerous advantages such as broad bandwidth, link security, instrument mass and power savings, and lower cost. Its applicability spans from the so-called last-mile short-distance connections to very long distances, such as with deep-space laser communication links. ${ }^{1,2}$ The last-mile communication instruments are available commercially from a number of suppliers. In optical intersatellite and satellite-to-ground links, there have recently been several milestone technology demonstrations. ${ }^{3-5}$ Despite the advantages of optical communication technology and the recent progress in this area, the atmospheric distortions and attenuation in the down- and uplinks, as well as very strong requirements for positioning and tracking of laser beams, have been major limiting factors, so far keeping this technology at the level of demonstrations. Thus, further developments of tracking techniques and exploratory searches for alternative ways of beam control and distortion compensation are crucial for the acceptance of this technology.

Recently, we have reported on our development of a nonlinear optical tracking technique based on the double phase-conjugation principle. ${ }^{6}$ This paper presents a differ-

$0091-3286 / 2008 / \$ 25.00$ ent approach. In essence, this approach uses a refractive index pattern induced in a layer of a nonlinear optical (NLO) material by the incoming optical beam to refract the outgoing optical beam. The outgoing-beam refraction is designed to provide for the beam-intensity distribution to have a sharp maximum at the communication counterpart location. Such a refractive index pattern, or simply a nonlinear lens, can be generated via any NLO mechanism that will lead to self-focusing or -defocusing, such as thermal, orientational, photorefractive, electronic, Kerr, or resonant. In dynamically changing situations, the lens is rewritten in different transverse locations on the NLO layer following the changes in the incoming beam angle. Thus, the outgoing beam intensity peak follows (tracks) the position of the distant source. In this paper, we describe this technique, the results of numerical modeling, and a proof-of-concept experiment using a liquid-crystal cell.

\section{Nonlinear Lens-Tracking Concept}

Communication between two optical terminals incorporating the NLO tracking system is depicted in Fig. 1. The optical signal (beam 1) from the fiber source of terminal 1 passes through the NLO system and is sent to a distant location where the second terminal (terminal 2) is expected to be. The NLO system is, essentially, a focusing optic (receiving lens, RL), and a layer of an NLO material placed 


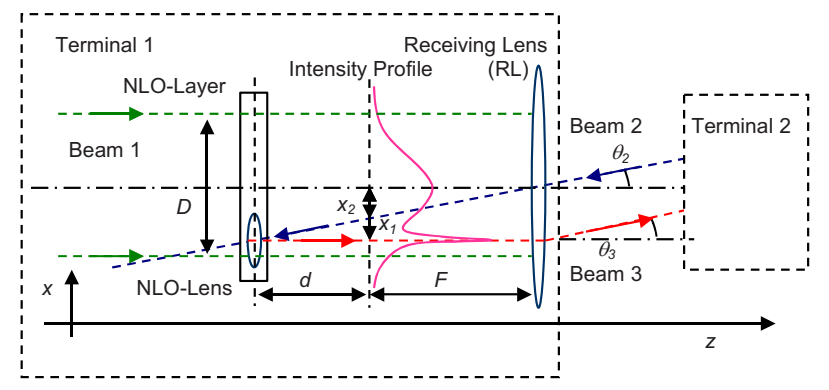

Fig. 1 Concept schematic of the tracking device (terminal 1) using a nonlinear focusing lens. Beam 1, the optical beam outgoing from terminal 1 towards a distant communicating party (terminal 2); beam 2 , a portion of the optical beam sent from terminal 2 and intercepted by the receiving lens (RL); beam 3 , a portion of beam 1 refracted by the NLO lens; $F$, the focal distance of the RL; $d$, the position of the NLO layer relative to the focal point of the RL.

close to the focal plane. The second terminal emits the optical signal (beam 2); a part of this signal is intercepted by terminal 1 and is focused onto the NLO layer. Due to the high optical nonlinearity of the NLO material, a nonlinear lens is induced. ${ }^{7}$ It should be noted here that we consider this concept to be applied to short-distance communication, where enough power can be intercepted to initiate the NLO effect. Let us consider a NLO material-with positive nonlinear coefficient causing self-focusing-placed behind the focal point of the receiving lens (RL, as shown in Fig. 1). The NLO material with a negative nonlinear coefficient causing self-defocusing should be placed before the focal point.

Let us assume that beam 2 comes to terminal 1 at a certain angle $\theta_{2}$ to the optical axis, which we consider to be small. The outgoing beam 1 is coaligned with the optical axis (the angle $\theta_{1}$ between beam 1 and the axis is equal to 0 ) and is collimated, having diameter $D$ on the left of RL (actually, $D$ can be much smaller than the RL aperture). We also assume that a thin NLO layer (of thickness $l$ ) is placed very close to the focal area of RL, and the nonlinearity is enough for beam 2 to induce a NLO lens with focal distance $f$ of the same order of magnitude as $d$ (the distance between NLO layer and RL focal point); thus the following is satisfied:

$l<d \ll F, \quad d \approx f$.

Under these conditions, the NLO lens is shifted perpendicularly to the optical axis by

$x_{1} \approx(F+d) \theta_{2}$.

This leads to focusing of some part of beam 1 in the focal area of the receiving lens (RL) (let us call this part beam 3) with about the same perpendicular shift. Consequently, the intensity distribution of beam 1 in the RL focal plane has a maximum at the transverse coordinate (beam 3) corresponding to the angular coordinate of terminal 2 . Then this maximum is projected by RL to the intensity maximum in the far field. Thus, a concentration of the power of the outgoing beam on the distant target is produced. Considering a moving target or moving host (terminal 2 or 1 , respectively), we can track its position by dynamic update of the NLO lens. The tracking speed in this case depends on the rate of NLO lens writing and decay. The field of view (FOV) of such a tracking system is limited by the diameter $D$ of the reading beam (beam 1) in the RL focal plane and the lens's focal distance $F$ :

$\mathrm{FOV} \approx D / F$

Here we have assumed that terminal 2 is located in the far field of terminal 1 and vice versa. At smaller distance between the terminals, the optimal layer position $d$ is dependent on that distance as the focal point of beam 2 becomes shifted further from RL.

A simple geometric consideration gives an estimate of the pointing precision of this tracking system. The angular pointing precision $\Delta \theta$ can be defined as the difference between the angle of beam $3\left(\theta_{3}\right)$ pointing to terminal 2 and the actual angular coordinate of this terminal $\left(\theta_{2}\right)$ :

$\Delta \theta=\theta_{3}-\theta_{2}$.

The major difference between these angles comes from the fact that the NLO lens is written by the beam propagating at the angle $\theta_{2}$ to the optical axis, but it is read by the beam propagating along the optical axis $\left(\theta_{1}=0\right)$. As a result, the peak of the intensity distribution of the reading beam is shifted from the axis by the almost $x_{1}$ in Eq. (2), and the peak of the incoming beam is shifted from the axis by

$x_{2}=F \theta_{2}$.

This leads to the tracking error

$$
\begin{gathered}
\Delta \theta=\frac{x_{1}-x_{2}}{F}=\frac{\theta_{2} d}{F}, \quad \text { resulting in } \quad \Delta \theta \ll \theta_{2} \\
\text { when } d \ll F .
\end{gathered}
$$

In the practical example of $F=5 \mathrm{~cm}, D=0.05 \mathrm{~cm}$, and $d=0.01 \mathrm{~cm}$, the relative angular error based on this estimate is $0.5 \%$ and the FOV is $10 \mathrm{mrad}$. Note that $x_{2}$ is always less than $x_{1}$, and $\Delta \theta$ is always positive. An additional NLO lens written by beam 1 can in part compensate this angular error. This NLO lens (larger in aperture) can additionally refract beam 3 toward the optical axis, reducing the difference between the coordinates $x_{1}$ and $x_{2}$.

\section{Theoretical Model of a Liquid-Crystal-Based System}

In order to obtain the qualitative picture of the suggested tracking effect, its generalized model was studied numerically using the diffraction-integral approach. The major goal of this modeling is to understand whether the NLO lens can be used for adaptive tracking with reasonable system parameters (laser power, material nonlinearity, etc.). This is judged by the far-field profile of the outgoing laser beam and the conditions and limitations of the system.

\subsection{Main Approximations}

The problem has been split into two steps: the first is the writing up of the NLO refractive index pattern (NLO lens) by laser beams 1 and 2 , and the second is the diffraction of beam 1 on this pattern. This corresponds to the thin-layer 


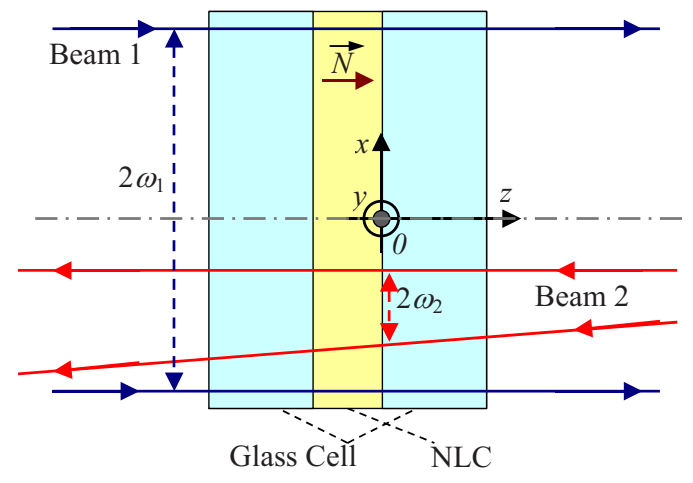

Fig. 2 Example of an NLO layer (nematic liquid crystal, NLC).

approximation, where the diffraction on the NLO lens does not affect its write-up process. Let us limit ourselves to the steady-state approximation with respect to the angles of the beams, where the NLO lens is written much faster than any change of angular directions of the beams. In other words, the angles $\theta_{1}$ and $\theta_{2}$ are constant during writing up and reading out.

We consider both beams ( 1 and 2 ) as Gaussian beams at the entrances to the tracking device, and then their propagation up to the NLO layer can be easily calculated. In a real situation, in fact, beam 2 would be truncated by the receive aperture (either a lens or a telescope), but we consider a general simplified system to avoid influence of the particularities of truncated-beam diffraction patterns on the result. Thus, the intensities of beams 1 and $2\left(I_{1,2}\right)$ can be expressed the following way at the left and right entrances of the NLO layer, respectively:

$$
\begin{aligned}
I_{1,2}(x, y, t)= & \frac{1}{2} \varepsilon_{0} c\left|E_{1,2}(x, y, t)\right|^{2}, \\
E_{1,2}(x, y, t)= & {\left[\frac{4 P_{1,2}(t)}{\pi \varepsilon_{0} c}\right]^{1 / 2} \frac{1}{\omega_{1,2}} \exp \left\{-\left(\frac{i \pi}{\lambda R_{1,2}}+\frac{1}{\omega_{1,2}^{2}}\right)\right.} \\
& \left.\times\left[\left(x-x_{01,02}\right)^{2}+\left(y-y_{01,02}\right)^{2}\right]\right\},
\end{aligned}
$$

where $E_{1,2}(x, y, t), P_{1,2}(t), \omega_{1,2}$, and $R_{1,2}$ are the electric field magnitudes, the powers, the radii, and the radii of curvature of beam 1 and beam 2 at the NLO layer entrances, respectively; $x_{01,02}$ and $y_{01,02}$ are the transverse coordinates of the centers of beams 1 and 2 , respectively; $\varepsilon_{0}$ is the vacuum permittivity; $c$ is the speed of light in vacuum; and $x$ and $y$ are the transverse coordinates.

\subsection{Liquid-Crystal Layer Description}

The NLO mechanism of the refractive index change can be of any origin: thermal, orientational, photorefractive, electronic, Kerr, resonant, etc. In the simplest case of an isotropic material or a crystal with a center of symmetry, the refractive index variations are mostly limited by the thirdorder nonlinearity. Let us consider the particular example that we used in our experiments: a thin layer of nematic liquid crystal (NLC) with thermal nonlinearity activated through absorption by dye doping (Fig. 2). The refractive index variations are described by the classical heatdiffusion equation (convection and boundary effects are neglected):

$\Delta n(x, y, z, t)=\frac{\partial n}{\partial T} \delta T(x, y, z, t)$,

$\left(\frac{\mathrm{d}}{\mathrm{d} t}-\hat{\kappa} \Delta\right) \delta T=\frac{1}{\rho C_{p}}\left[\alpha_{1} I_{1}(x, y, z, t)+\alpha_{2} I_{2}(x, y, z, t)\right]$,

where $\Delta n(x, y, z, t)$ is the refractive-index change; $(\partial n / \partial T)$ is the thermal nonlinearity coefficient, $\delta T(x, y, z, t)$ is the temperature variation produced by the absorption of the laser beams, $\hat{\kappa}$ is the thermal conductivity tensor, $\rho C_{p}$ is the specific heat, $\alpha_{1,2}$ are the absorption coefficients for the beams (these coefficients may be different for beam 1 and beam 2 if they have different wavelengths), and $I_{1,2}(x, y, z, t)$ are the intensities of the laser beams.

Solution of Eq. (8) in general form is very difficult; thus we limit ourselves to the particular example of transient formation of refractive index and temperature patterns. In other words, we deal with laser pulses with duration $\tau_{P}$ shorter than the temperature pattern's relaxation time:

$\tau_{P}<\tau_{1,2}=\frac{1}{\kappa_{\|} l^{-2}+\kappa_{\perp} \pi \omega_{1,2}^{-2}}$,

where $\tau_{1,2}$ are the refractive index pattern relaxation times for beams 1 and 2, respectively; $l$ is the NLC layer thickness; and $\kappa_{\perp}$ and $\kappa_{\|}$are the thermal conductivities perpendicular and parallel to the NLC director ( $\mathbf{N}$ in Fig. 2). ${ }^{8}$ We also assume the layer thickness and absorption are very small, so that both beams' intensities are constant along the $z$ direction. Under these approximations we neglect the second term in the thermal equation (8) and, assuming a square pulse shape, obtain the following expression for the refractive index change at the end of the laser pulse (at $\left.t=\tau_{P}\right)$ :

$\Delta n(x, y)=\left(\frac{\partial n}{\partial T}\right) \frac{\tau_{P}}{\rho C_{p}}\left[\alpha_{1} I_{1}(x, y)+\alpha_{2} I_{2}(x, y)\right]$,

where $I_{1}(x, y)$ and $I_{2}(x, y)$ are defined by Eq. (7). Further, we assume the absorption coefficients equal for the two beams $\left(\alpha_{1}=\alpha_{2}=\alpha\right)$. Under these circumstances the refractive index pattern is given by the sum of the intensity distributions of the laser beams, and in fact serves as a phase mask for beam 1 and beam 2. A similar structure of the refractive index pattern should occur for any local mechanism of nonlinearity where the spatial transfer mechanisms (diffusion, drift, convection, etc.) are negligible.

\subsection{Diffraction-Integral Approach}

We focus our analysis on the effect on beam 1 . The intensity and electric field magnitude distribution of beam 1 after the passage through the NLO layer becomes too complicated for analytical treatment; thus we took a numerical approach. The electric field amplitude of beam $1, E_{1}\left(\mathbf{r}_{2}\right)$ at position $\mathbf{r}_{2}=\left(x_{2}, y_{2}, z_{2}\right)$ can be calculated at any distance $z_{2}$ 


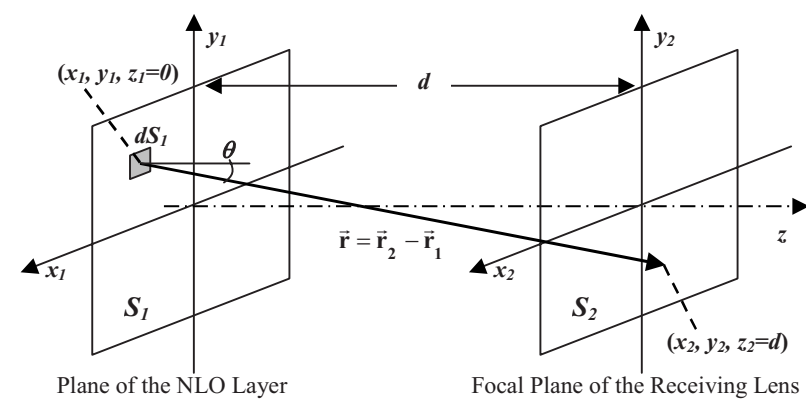

Fig. 3 Calculation geometry for the Fresnel-Kirchhoff diffraction integral.

(in the plane $S_{2}$ ) from the NLO layer $\left(S_{1}\right)$ using well-known Fresnel-Kirchhoff diffraction integral (Fig. 3): ${ }^{9}$

$$
\begin{aligned}
E_{1}\left(\mathbf{r}_{2}\right)= & \frac{i}{2 \lambda_{1}} \iint_{S_{1}} E_{1}\left(x_{1}, y_{1}\right) \frac{1+\cos \theta}{r} \\
& \times \exp \left\{-i k_{1}\left[r+l \cdot \Delta n\left(x_{1}, y_{1}\right)\right]\right\} \mathrm{d} S_{1},
\end{aligned}
$$

$r=\left|\mathbf{r}_{2}-\mathbf{r}_{1}\right|$,

where $\lambda_{1}$ is the wavelength of beam 1 and $k_{1}=2 \pi / \lambda_{1}$ is its wave number, $\mathrm{d} S_{1}$ represents the element of surface on the plane just after the NLO layer with coordinates $\mathbf{r}_{1}=\left(x_{1}, y_{1}, z_{1}=0\right), \Delta n\left(x_{1}, y_{1}\right)$ and $E_{1}\left(x_{1}, y_{1}\right)$ are described by Eqs. (10) and (7), respectively, and $\theta$ is the angle between the propagation direction $\mathbf{r}$ and the normal to the plane $S_{1}$.

We calculated numerically the electric field distribution $E_{1}\left(x_{2}, y_{2}\right)$ and the intensity $I_{1}\left(x_{2}, y_{2}\right)$ of beam 1 for the output plane $S_{2}$ representing the focal plane of the receiving lens (RL). From this we are able to evaluate qualitatively the angular distribution $I_{1 F F}\left(\theta_{1 x}, \theta_{1 y}\right) \propto I_{1}\left(x_{2} / F, y_{2} / F\right)$ of beam 1 (containing beam 3 ) in the far field after RL, assuming its property of projecting the image from the focal plane to the far field.

The quantitative assessment of the tracking capability is done by calculating the portion of beam 1 reaching the distant receiver. To do so we calculate the weighted projection of the output beam electric field (with and without NLO pattern) on the electric field of the input beam, $E_{2}\left(x_{2}, y_{2}\right)$. This weighted projection reaches its maximum value of 1 when beam 2 is phase-conjugate to beam 1 :

$C_{1,0}=\frac{\left|\iint_{S_{2}} E_{2}\left(x_{2}, y_{2}\right) \cdot E_{1,01}\left(x_{2}, y_{2}\right)^{*} \mathrm{~d} x_{2} \mathrm{~d} y_{2}\right|^{2}}{\iint_{S_{2}} E_{2}\left(x_{2}, y_{2}\right) \cdot E_{2}\left(x_{2}, y_{2}\right)^{*} \mathrm{~d} x_{2} \mathrm{~d} y_{2} \cdot \iint_{S_{2}} E_{1,01}\left(x_{2}, y_{2}\right) \cdot E_{1,01}\left(x_{2}, y_{2}\right)^{*} \mathrm{~d} x_{2} \mathrm{~d} y_{2}}$,

where $C_{1}$ and $C_{0}$ are the coupling coefficients with and without the NLO lens induced by input beam 2, $E_{1}\left(x_{2}, y_{2}\right)$ and $E_{01}\left(x_{2}, y_{2}\right)$ are the outgoing electric field distributions with and without input beam 2, and the integration area $S_{2}$ is the total area of the beams in the RL focal plane. The indicator of the tracking system performance is the ratio $R$ of those coupling coefficients (coupling ratio):

$R=C_{1} / C_{0}$.

It shows how much increase of the signal at the receiving end we may achieve by use of the NLO tracking system.

\subsection{Results of Numerical Modeling}

Some examples of the calculated intensity distributions of beam 1 and beam 2 in the focal plane of the receiving lens (RL) are shown in the Fig. 4(a)-4(d). When there is no input beam $\left(P_{2}=0\right)$, beam 1's intensity distribution is a smooth, close to Gaussian profile [dotted curves in Fig. 4(a) and 4(b)]. Its width in the focal plane determines the farfield divergence and field of view of the optical system as defined in Eq. (3). In this example beam 1 is collimated and covers a large area in the far field. When beam 2 is present [its focal plane intensity distribution is given by the dashed curves in Fig. 4(a) and 4(b)], the peak of intensity appears on beam 1 at the same transverse position as on beam 2 [solid curves in Fig. 4(a) and 4(b), and 3-D distributions in
Fig. 4(c) and 4(d)]. This maximum represents the narrowdivergence portion of beam 1 after RL, which is also called beam 3 in Fig. 1. The width of this maximum depends on the material nonlinearity, beam 2 power, and NLO layer position. The calculation parameters are $P_{1}=200 \mathrm{~mW} ; P_{2}$ $=1 \mathrm{~mW} ; \tau_{\mathrm{p}}=1 \mathrm{~ms} ; F=0.8 \mathrm{~cm}, d=35 \mu \mathrm{m} ; \omega_{1}=0.017 \mathrm{~cm}$ at the focal point; $\omega_{2}=0.2 \mathrm{~cm}$ in front of the lens (input); $\partial n / \partial t=5 \times 10^{-4} ; \rho C_{p}=1.5 \mathrm{~J} \mathrm{~K}^{-1} \mathrm{~cm}^{-3} ; l=25 \mu \mathrm{m} ; \alpha_{1}=\alpha_{2}$ $=44 \mathrm{~cm}^{-1} ; \lambda=1.5 \mu \mathrm{m} ; \quad \kappa_{\perp} \approx 0.8 \times 10^{-3} \mathrm{~cm}^{2} \mathrm{~s}^{-1} ; \quad$ and $\kappa_{\|}$ $\approx 1.25 \times 10^{-3} \mathrm{~cm}^{2} \mathrm{~s}^{-1}$; the figures are given for a 4-n-pentyl-4' -cyanobiphenyl (5CB) NLC. ${ }^{8}$ In this particular case, the width of beam 1's maximum is close to that of beam 2, signifying that its far-field spot size is close to diffraction-limited (because the spot size of beam 2 is diffraction-limited).

In fact, the NLO lens has concentrated the power of beam 1 into the less divergent beam 3 . The nonzero input angle of beam 2 leads to a shift of its maximum in the RL focal plane [dashed curve in Fig. 4(b)]. Approximately the same shift is observed for the maximum of beam 1 (beam 3); see the solid curve in Fig. 4(b). Any possible mismatch of the beam 2 and beam 3 positions (tracking error) is much smaller than the diameters of the beams.

The amplitude of the intensity peak (beam 3 ) exceeds by several times the initial intensity of beam 1 in that direction. It depends on the parameters of the material, the laser 


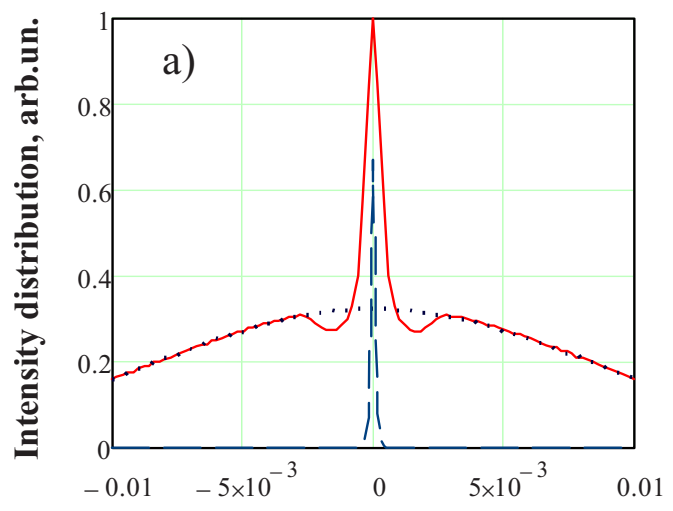

Transverse spatial coordinate $(x), \mathrm{cm}$

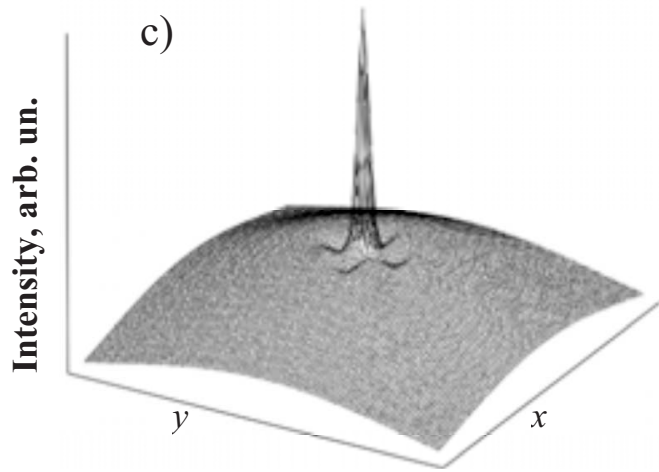

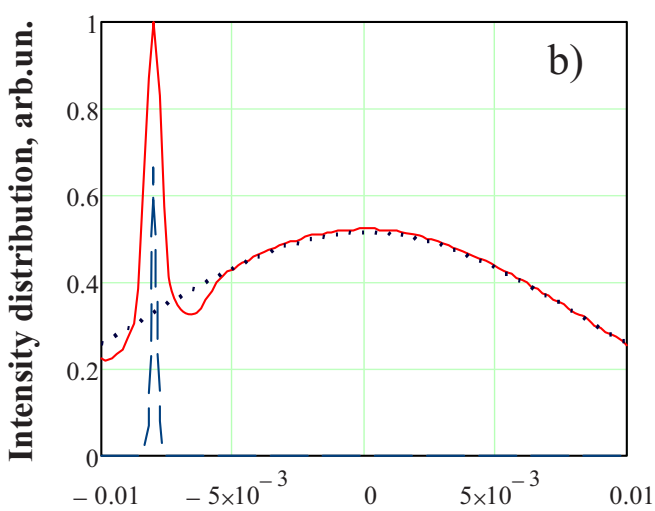

Transverse spatial coordinate $(x), \mathrm{cm}$

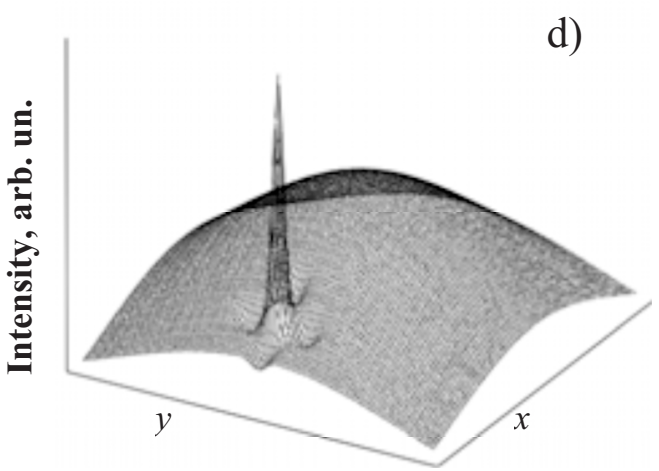

Fig. 4 Laser intensity distributions at the focal plane of the receiving lens (RL). Solid curves on graphs (a), (b) and in the 3-D plots are for beam 1 calculated by the Fresnel integral (11). Dotted curves are for beam 1 in the absence of beam 2, and dashed curves beam 2; both are calculated for the Gaussian beam propagation (7). (a) Along $x$ axis at $\theta_{2 x}=0, \theta_{2 y}=0$; (b) along $x$ axis at $\theta_{2 x}=10 \mathrm{mrad}, \theta_{2 y}=0$; (c) in the $(x, y)$ plane at $\theta_{2 x}=0, \theta_{2 y}=0 ;(d)$ in the $(x, y)$ plane at $\theta_{2 x}=10 \mathrm{mrad}, \theta_{2 y}=0$.

beams, the RL focal distance, and the NLO cell position. Figure 5 shows some of the key dependences of the coupling ratio [defined by Eqs. (12) and (13)]. In the absence of input beam 2, the coupling ratio is equal to one (i.e., there is no nonlinear effect) [Fig. 5(a)]. At small input power of beam 2 , the coupling ratio grows linearly in spite of the fact that it is a NLO effect driven by the power of beam 2. At some point the coupling ratio reaches a maximum and then decreases as the NLO lens becomes too strong. The coupling ratio can be optimized by choosing the position of the NLO cell with respect to the RL focal plane [Fig. 5(b)]. The smaller the input beam 2 power, the closer to the focal point the NLO cell should be placed. The improvement of the signal at the receiving point (terminal 2 ) is expected to be as much as an order of magnitude.

\section{Proof-of-Concept Experiment}

The goal of this experiment is to demonstrate the qualitative correspondence of experiment to the theory and assess the tracking capabilities of the NLO tracking system.

\subsection{Experimental Setup}

The tracking test bed consists of two communicating counterparts (Fig. 6); each of them includes a 1.5- $\mu \mathrm{m}$-wavelength laser (coupled to a single-mode fiber), detector, and polarization controller. One of them (terminal
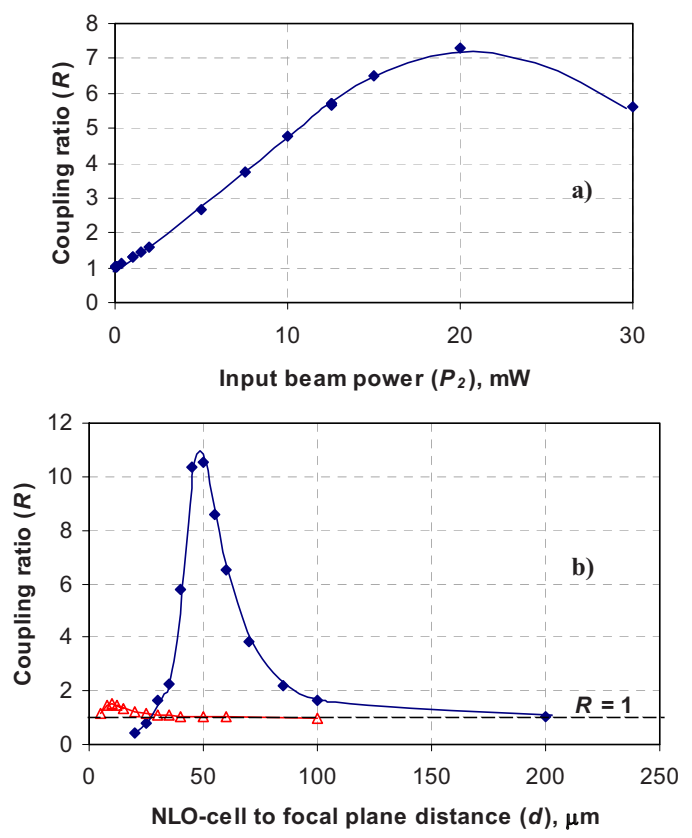

Fig. 5 Coupling ratio (a) versus input beam power and (b) versus distance between NLO cell and focal plane of the RL. (a) $d=35 \mu \mathrm{m}, \tau_{p}=0.2 \mathrm{~ms}$; (b) $\tau_{p}=0.2 \mathrm{~ms}, P_{2}=50 \mathrm{~mW}$ for diamonds, and $P_{2}=0.5 \mathrm{~mW}$ for triangles; the dashed line corresponds to the case of no NLO contribution $(R=1)$. 


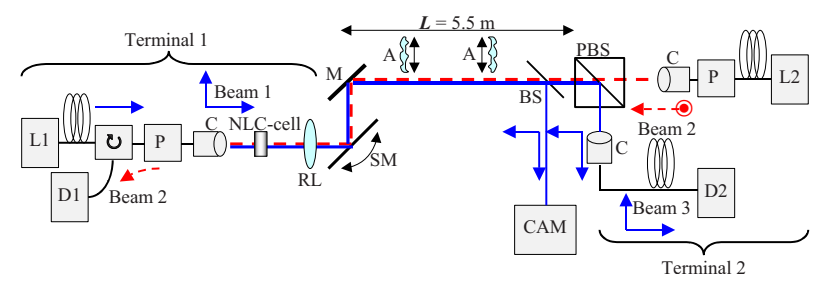

Fig. 6 Schematic of NLO tracking system experiment. Solid lines and arrows show the path and direction of beam 1; dashed lines and arrows show the path and direction of beam 2. Notation L1,2, lasers; D1,2, detectors; $\circlearrowleft$, circulator; P, polarization controllers; C, couplers based on GRIN lenses (0.5-mm aperture); RL, receiving lens (2-cm aperture, $4.5-\mathrm{cm}$ focal distance); SM, steering mirror; $\mathrm{M}$, mirror; $\mathrm{A}$, aberration plates; BS, beamsplitter; PBS, polarization beamsplitter; CAM, infrared camera.

1) includes the NLO tracking system (NLC cell and a lens); the other (terminal 2) is a passive terminal. The scanning mirror (SM, based on a voice coil) located in front of terminal 1 provides simulation of jitter in horizontal and vertical directions (it is not a part of the tracking system). The tracking system's performance is evaluated by its ability to point the laser beam to the fiber coupler at the other side.

Beam 2 is emitted from the fiber optic coupler based on a gradient index (GRIN) lens with $\sim 0.5$-mm aperture. Its polarization is set to horizontal by the polarization controller, to pass through the polarization beamsplitter (PBS) with minimal losses. With its diffraction-limited divergence, beam 2 reaches terminal 1, $5.5 \mathrm{~m}$ away. About $30 \%$ of beam 2's power is intercepted by the input aperture of terminal 1, a receiving lens (RL) with focal distance $4.5 \mathrm{~cm}$ and clear aperture $2 \mathrm{~cm}$. The input beam 2 is focused on the NLO layer located close to the RL focal plane.

As the NLO material we chose the NLC 4- $n$-pentyl-4' -cyanobiphenyl (5CB) doped with infraredabsorbing dye. The NLC layer has slowly varying thickness along transverse coordinate (accomplished by use of different thickness spacers on the two sides). Homeotropic orientation of the NLC is achieved by coating the cell walls with hexadecyltrimethyl ammonium bromide (HTAB). The thermal nonlinearity caused by the change of order parameter of homeotropically aligned 5CB is positive, in agreement with our model.

The collimated beam 1 with radius $\omega_{1}=0.017 \mathrm{~cm}$ is emitted by the fiber coupler and intersected with beam 2 inside the NLC cell, as shown in Fig. 2. The vertically polarized beam 1 is reflected from the polarization beamsplitter with minimal losses after passage through the 5.5-m distance. In the absence of beam 2 , beam 1 covers an area of much larger diameter (several centimeters) than the aperture of the receiving fiber coupler (identical in type and position to the transmitting coupler). The received portion of beam 1 is in the range of $0.1 \%$ to $0.2 \%$ of its total power and is monitored by detector D2. The transverse intensity profile of beam 1 in front of the receiving end is monitored by the IR camera.

\subsection{Study of the Nonlinear Lens}

When beam 2 is present, it modulates the transverse profile of beam 1 through the formation of an NLO lens. This results in the appearance of a narrow-angle component in
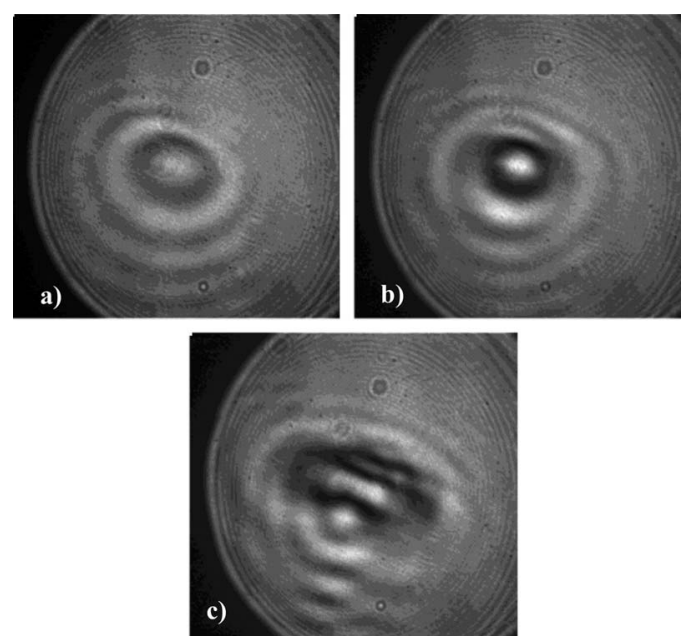

Fig. 7 The intensity profiles (gray scale images) of the beam from the tracking terminal 1 at the plane of the passive terminal fiber coupler at different power levels of beam $2\left(P_{2}\right)$ intercepted by terminal 1: (a) $16.6 \mathrm{~mW}$; (b) $24.2 \mathrm{~mW}$; (c) $48 \mathrm{~mW}$. Beam 1 is $\mathrm{cw}, P_{1}$ $=8.3 \mathrm{~mW}, I=18.7 \mu \mathrm{m}$. The bright central spot corresponds to the narrow-angle reverse-directed signal.

beam 1 (beam 3) with position matching the position of the distant transmitting and receiving aperture [Fig. 7(a) to $7(\mathrm{c})]$. With increase in the power of beam 2, one may see the slight increase in intensity at the center [Fig. 7(a)], then the formation of the bright spot [Fig. 7(b)], and, when the power exceeds the optimum, significant aberrations [Fig. $7(\mathrm{c})]$.

In most of our experiments beam 2 is modulated with pulse duration 0.1 to $1 \mathrm{~ms}$ and repetition rate 10 to $1000 \mathrm{~Hz}$, and beam 1 is continuous wave. This allows monitoring the dynamics of the refractive index lens induced in the NLO material. The dynamics of the coupling ratio, which is essentially the received signal normalized to the signal in the absence of beam 2, is shown in Fig. 8 for different intercepted powers of beam 2 at terminal 1 . At small input power (when $R<1.2$ ) the refractive index lens

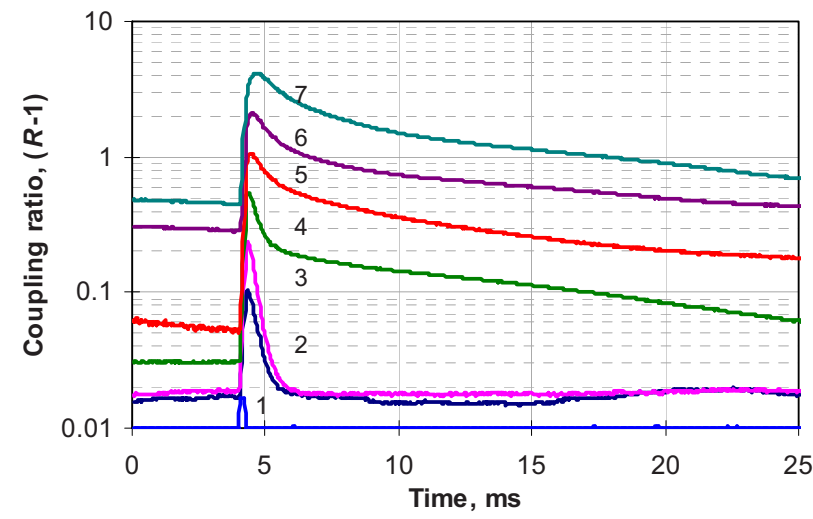

Fig. 8 Dynamics of the reverse-directed signal: oscilloscope traces (curves 2 to 7 ) of the signal received from detector D2 calibrated to provide the coupling ratio $(R-1)$ for different powers of beam 2 intercepted by terminal $1(7.5,11.3,14,16.6,20.4,30.4 \mathrm{~mW}$, respectively). Curve 1 is a trace for beam $2\left(\tau_{P}=0.2 \mathrm{~ms}\right.$ at $\left.20 \mathrm{~Hz}\right)$; beam 1 is $\mathrm{cW}, P_{1}=8.3 \mathrm{~mW}, l=18.7 \mu \mathrm{m}$. 
has a decay time of about $1 \mathrm{~ms}$, which corresponds to the decay of a thermal pattern of the size of beam 2 in the RL focal area [as defined in Eq. (9)]. At larger power, the decay time increases to several milliseconds. The NLO lens does not vanish completely in the period between pulses $(50 \mathrm{~ms}$ for $20-\mathrm{Hz}$ rate), thus producing some pulse-after-pulse NLO lens accumulation (see the growth of the baseline in Fig. 8). This can be a result of two factors. The first is that a much larger area of the NLO layer is involved in the formation of the NLO lens because of diffusive thermal spread when the laser beam supplies enough power. This is very important, since it allows one to concentrate the power from the larger area and achieve efficiency $R \gg 1$, but at the same time it limits the tracking response time. The second factor is the contribution of orientational nonlinearity with a typical response time of 50 to $100 \mathrm{~ms}$.

The slowly varying thickness of the NLC layer allows us to see the effect of the cell thickness on the transmitted signal [Fig. 9(a)]. With the cell thickness change from 5 to $20 \mu \mathrm{m}$ the exponential absorption factor $(\alpha l)$ changes from 0.11 to 0.5 (curve 2), leading to an increase in system efficiency $R$ from 2 to 5 (curve 1 ).

The experimental coupling ratio versus the beam 2 power intercepted by terminal 1 is shown in Fig. 9(b). It shows a lot of similarities with the numerical modeling [Fig. 5(a)], such as reaching the maximum of $R=4$ to 6 at beam 2 power of $20 \mathrm{~mW}$ [see the maximum of curve 1 in Fig. 9(b)]. Note the major difference: The experimental dependence is not linear at small powers. This may be explained by the strong dependence (growth) of the NLO coefficient of the NLC on approaching the temperature of the phase transition from nematic to isotropic liquid $\left(350^{\circ} \mathrm{C}\right)$. Noticeably, curve 4 shows higher efficiency and sensitivity than curve 3 [Fig. 9(b)], just due to the increase of beam 1 power leading to slight growth of the working temperature of NLC.

The modeling was done for the transient scenario of NLO lens formation, where its profile repeats the profile of the laser beam. Thus, the modeling results may not be comparable with traces 2 to 4 [Fig. 9(b)] measured at continuous wave. Since in those cases the NLO lens is written during a longer time, it provides better sensitivity. As may be seen, at the $2-\mathrm{mW}$ level the received signal on utilizing the NLO lens is twice as large as without it $(R-1 \approx 1$, trace $4)$. It may be further suggested that if the NLO tracking system is used at both ends, then the signal growth due to even modest $R \approx 1.1$ will be enough to trigger side-by-side coupling efficiency growth to the saturation level $(R \approx 4$ to $6)$. The modeling of this process and its experimental study lie out of the scope of this paper. In the future, we believe it is realistic to hope for such a system with sensitivity in the microwatt range.

We see a strong dependence of the coupling ratio on the $z$ position of the NLC cell [Fig. 9(c)], very similar to the theoretical one calculated for the same numerical aperture of the focusing lens $(\mathrm{RL})$ (though scaled down $\approx 4$ times) [Fig. 5(b)]. The differences in position of the maximum may be also attributed to the difference of the transverse profile of beam 2 in the experiment from the Gaussian model. The input aperture (RL) truncates the Gaussian beam 2 and focuses it on the NLC cell; therefore, the way
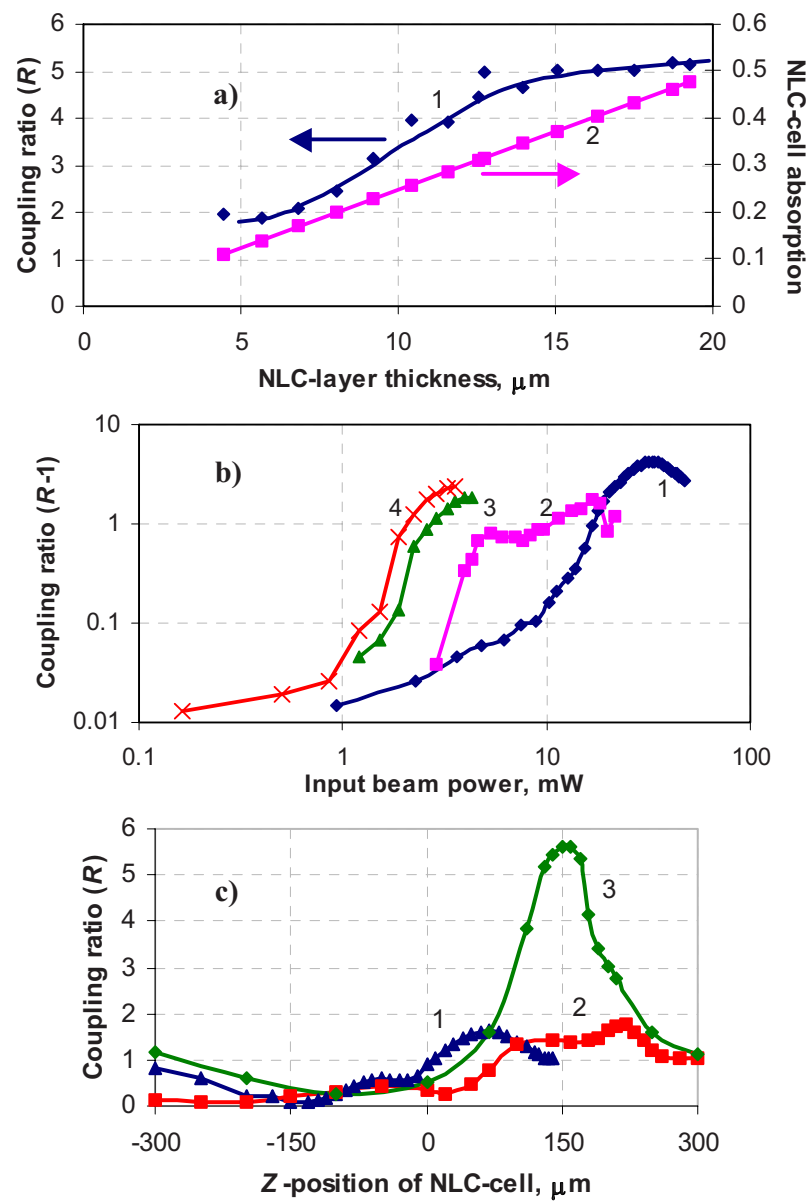

Fig. 9 The experimental coupling ratio $(R$ and $R-1)$ versus the following parameters: (a) NLC layer thickness I, (b) beam 2 power intercepted by terminal 1 , and (c) $z$ position of NLC cell. (a) $P_{1}=8.3 \mathrm{~mW}, P_{2}=48 \mathrm{~mW}, \tau_{P}=0.1 \mathrm{~ms}$, rate $20 \mathrm{~Hz}$; (b) curve 1: $P_{1}=8.3 \mathrm{~mW}, \quad \tau_{P}=0.2 \mathrm{~ms}$, rate $20 \mathrm{~Hz}, \quad l=18.7 \mu \mathrm{m}$; curve 2: $P_{1}=8.3 \mathrm{~mW}, I=4.5 \mu \mathrm{m}$; curve $3: P_{1}=8.3 \mathrm{~mW}, I=14 \mu \mathrm{m}$; curve 4 : $P_{1}=15.2 \mathrm{~mW}, I=14 \mu \mathrm{m}$; (c) curve 1: $P_{1}=15.2 \mathrm{~mW}, P_{2}=13.3 \mathrm{~mW}$, $\tau_{P}=0.1 \mathrm{~ms}$, rate $20 \mathrm{~Hz} ; \quad l=5 \mu \mathrm{m} ;$ curve $2: P_{1}=15.2 \mathrm{~mW}, P_{2}$ $=48 \mathrm{~mW}, \tau_{P}=0.1 \mathrm{~ms}$, rate $20 \mathrm{~Hz}, l=5 \mu \mathrm{m}$; curve $3: P_{1}=8.3 \mathrm{~mW}$, $P_{2}=48 \mathrm{~mW}, \tau_{P}=0.1 \mathrm{~ms}$, rate $20 \mathrm{~Hz}, l=18.7 \mu \mathrm{m}$. Beam 1 is $\mathrm{cw}$; pulse duration and rate are shown for beam 2 (if not otherwise specified, beam 2 is $\mathrm{cw}$ ).

the intensity profile of the intercepted part of beam 2 changes along the $z$ axis is different from the Gaussian model.

\subsection{Tracking of Angular Disturbances}

The formation of a narrow-angle component in the beam 1 intensity profile, which is reverse-directed to the input beam, is also observed when angular disturbance is introduced. The steering mirror is used to disturb the angular position of beam 2 with controlled frequency and amplitude. The nontracking portion of beam 1 follows this angular modulation at the receiving end, while the tracking portion (beam 3) stays at the position of the receiving aperture regardless of disturbances. The screenshots of beam 1 in front of the receiving aperture for angular disturbances of different frequency and peak-to-peak amplitude in the $x$ and $y$ directions are shown in Fig. 10(a)-10(c). The disturbances were simultaneously applied along the $x$ and $y$ axes 

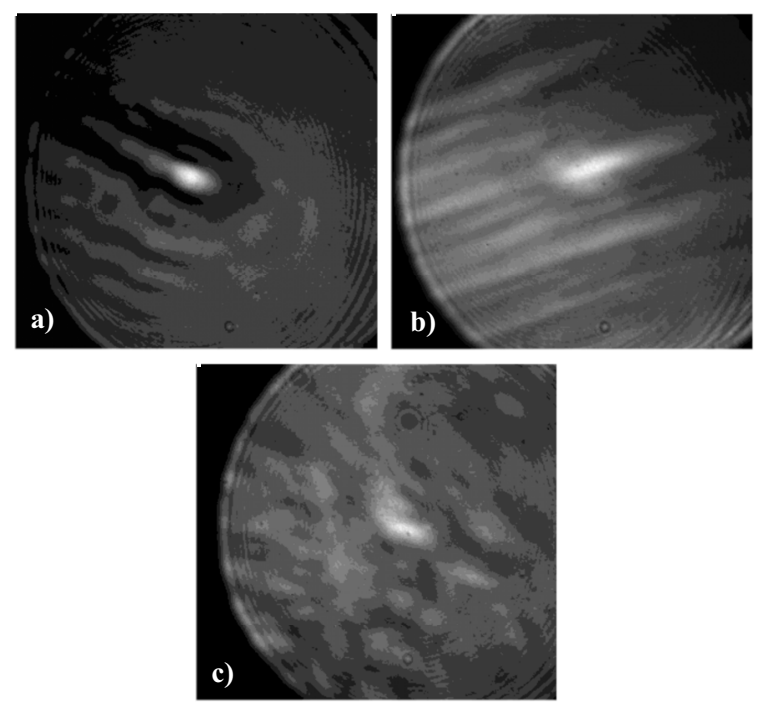

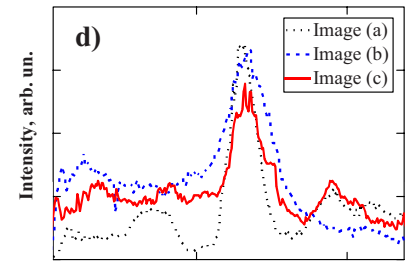

Transverse coordinate $(x)$, arb. un

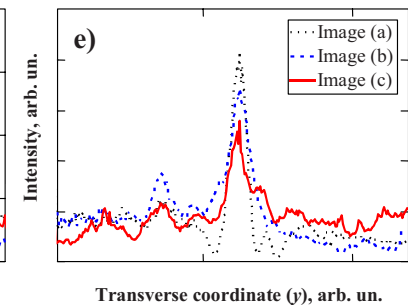

Fig. 10 Gray-scale screenshots [(a) to (c)] and corresponding intensity profiles $[(\mathrm{d})$ to $(\mathrm{e})]$ of the beam from the tracking terminal 1 at the plane of the passive terminal fiber coupler while tracking angular disturbances of different frequency and peak-to-peak amplitude in the $x$ and $y$ direction $\left(f_{\theta x}, f_{\theta y}, \theta_{2 x}\right.$, and $\left.\theta_{2 y}\right)$ : (a) $f_{\theta x}=0.5 \mathrm{~Hz}, f_{\theta y}$ $=0.73 \mathrm{~Hz}, \theta_{2 x}=5.2 \mathrm{mrad}, \theta_{2 y}=4.2 \mathrm{mrad}$; (b) $f_{\theta x}=10.5 \mathrm{~Hz}, f_{\theta y}=13 \mathrm{~Hz}$, $\theta_{2 x}=4.2 \mathrm{mrad}, \theta_{2 y}=5.2 \mathrm{mrad}$; (c) $f_{\theta x}=154.7 \mathrm{~Hz}, f_{\theta y}=220 \mathrm{~Hz}, \theta_{2 x}$ $=3.1 \mathrm{mrad}, \theta_{2 y}=3.1 \mathrm{mrad}$. Experimental parameters for (a) and (b): Beam 1: $P_{1}=8.3 \mathrm{~mW}, \mathrm{cw}$; beam 2: $P_{2}=11 \mathrm{~mW}, \tau_{P}=0.5 \mathrm{~ms}$, rate $300 \mathrm{~Hz}, \quad I=18.7 \mu \mathrm{m}$. For (c): $P_{1}=8.3 \mathrm{~mW}, P_{2}=15.3 \mathrm{~mW}$; both beams are modulated with $\tau_{P}=0.2 \mathrm{~ms}$ and rate $180 \mathrm{~Hz} ; l=18.7 \mu \mathrm{m}$.

at similar frequencies and amplitudes. At low frequency $(<1 \mathrm{~Hz})$ an image close to the nondisturbed case is observed. At higher frequency $(\approx 10 \mathrm{~Hz})$ a significant tail of the central beam 3 is observed with direction corresponding to the direction of the disturbance. This means that while the NLO lens is moving from spot to spot in the NLC layer following the disturbance, it does not disappear fast enough from the previous spots. Thus, refraction by outdated, not yet vanished patterns forms this tail. For the 2-D disturbance in the frequency range of $200 \mathrm{~Hz}$ (beyond which voice-coil steerers usually do not perform well) the central tracking spot still shows up, providing signal improvement by 2 to 3 times. Similar improvement and a narrow-angle spot are observed for linear (1-D) disturbances of $500 \mathrm{~Hz}$. However, high-frequency observations are successful only when both beams are pulsed (no readout is happening between pulses). Figure 10(d) and 10(e) show the intensity distributions in $x$ and $y$ through the center of the screen on the images in Fig. 10(a) to 10(c). The central peak shows several fold signal improvement for all angular disturbances.

When the input beam's angular position deviates from the center, the tracking efficiency decreases due to the
Gaussian profile of the readout beam (beam 1). In our experiments the tracking unit produced considerable signal improvement within the angular span of \pm 4 mrad (falling to $R=1.5$ at the edges), resulting to $\mathrm{FOV} \approx 8 \mathrm{mrad}$. This is in agreement with the projected FOV $\approx 7.6 \mathrm{mrad}$ as estimated by Eq. (3). A larger FOV is possible with a shorterfocal-distance lens or a wider readout beam.

\section{Compensation of Wavefront Distortions}

Thus it has been demonstrated that the reverse-directed beam is formed within the field of regard at any angle of incidence of the input beam. Let us assume that several beams are set to input the NLO tracking device at different angles. Since each of the beams forms a NLO lens in its own location in the NLC layer, they do not interfere with each other and can result in simultaneous formation of several reverse-directed beams from one readout beam. Such a situation may be realized when we want to broadcast the signal to several terminals, or when we deal with distorted beams. In the latter case, the input beam may be represented by a speckled angular structure at the receiving lens. ${ }^{10}$ Then, each major speckle produces a corresponding focusing microlens. The multitude of those microlenses forms a pattern of refractive indices carrying the information of the speckle field. Such a refractive index pattern modulates the readout beam, strengthening components reverse-directed to the major speckles. After passing back through the distortions one may expect a stronger signal to reach the receiver because of partial compensation of the distortions.

We tested the feasibility of such a scenario in our experiment by inserting two aberration plates (simultaneously) in the beam path between the terminals (Fig. 6). The use of two aberration plates at different places allows better representation of aberrations extending along the transmission path (like those happening in a long atmospheric path) and ensures that the adaptive capability does not critically depend on the position of the aberration plate. The speckle structures of the beams entering two terminals (in the absence of a NLO pattern) are shown in Fig. 11(a) and 11(b). One can notice very strong aberrations.

When both beams are enabled, resulting in NLO pattern formation, the experimental observations show clear and stable formation of a reverse-directed beam having close to Gaussian shape after passage back through two aberration plates [Fig. 11(c)]. If angular disturbance is introduced by the beamsteerer (sinusoidal scanning along both $x$ and $y$ axes with SM in Fig. 6) at fixed aberration-plate positions, the reverse-directed beam stays for jitter frequencies more than $100 \mathrm{~Hz}$ in both $x$ and $y$ directions [Fig. 11(d)]. The appearance of the reverse-directed beam was accompanied by a 3 - to 5-fold increase of the signal received on the fiber-coupled detector, corresponding to the factor $R=3$ to 5 . In addition to scanning the beam at fixed aberration-plate positions, we also tested what happens when the plates are moved manually. Shifts of aberration plates can change the $R$ factor within its range, but do not critically affect the observation: the reverse-directed beam stays.

The interesting result with aberration plates is that the reverse-directed beam profile is much closer to the original Gaussian beam than in the experiments without the aberration plates [compare transverse profiles in Fig. 11(e) and 

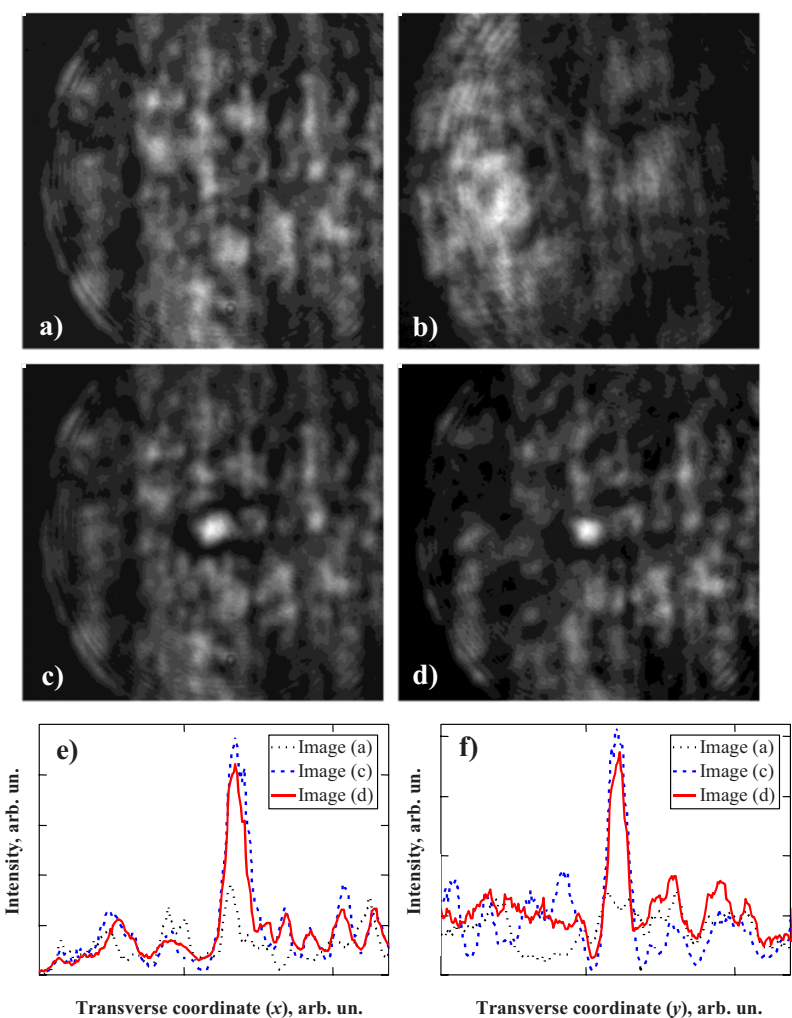

Fig. 11 Grayscale screenshots [(a) to (c)] and corresponding intensity profiles $[(d)$ to $(e)]$ of the beams from both terminals in the presence of two aberration plates with and without angular disturbances: (a) a screenshot of beam 1 in front of terminal 2 in the absence of beam 2 with no angular disturbances; (b) a screenshot of beam 2 in front of terminal 1 in the absence of beam 1 with no angular disturbances; (c) a screenshot of beam 1 in front of terminal 2 with beam 2 present and no angular disturbances; (d) a screenshot of beam 1 in front of terminal 2 with beam 2 present and with angular disturbances: $f_{\theta x}=100.7 \mathrm{~Hz}, f_{\theta y}=111 \mathrm{~Hz}, \theta_{2 x}=3.1 \mathrm{mrad}$ $\theta_{2 y}=3.1 \mathrm{mrad}$; (e), (f) intensity profiles along the $x$ and $y$ axes corresponding to the preceding images. Experimental parameters: $P_{1}$ $=8.3 \mathrm{~mW}, P_{2}=48 \mathrm{~mW}$; both beams are modulated with $\tau_{P}=0.2 \mathrm{~ms}$ and rate $180 \mathrm{~Hz} ; l=18.7 \mu \mathrm{m}$.

11(f) with those in Fig. 10(d) and 10(e)]. This suggests that the NLO tracking device modulates the transverse structure of the readout beam so that a considerable part of it is in fact phase-conjugate to the original input beam. The presence of two aberration plates allows spatial filtering of the phase-conjugate component. This results in observation of a cleaner reverse-directed beam than in the case without aberrators. Of course, detailed modeling and a separate dedicated set of experiments will have to be performed to rigorously prove the phase-conjugation function of the system we study. This topic is out of the scope of this paper and will be explored in further work.

\section{Discussion}

Let us consider application of the described concept to a typical short-distance, ground-to-ground communication link (last-mile connection).

In a typical arrangement, two identical optical terminals are located $1 \mathrm{~km}$ apart. The receiver aperture diameter is determined by the requirement to collect as much light as possible and to reduce atmospheric scintillations by aver- aging over the aperture. Typically, that diameter can be about $15 \mathrm{~cm}$. The transmitted beam divergence is about $2 \mathrm{mrad}$. This kind of system is not expected to have any tracking capability, for the communicating points are not moving, and the beam divergence covers small angular disturbances. High output power, which is typically in the range of $0.5 \mathrm{~W}$ (routinely achieved with fiber amplifiers or by combining a few lasers of the same wavelength), is capable of compensating for free-space losses. Using a classical tracking system could improve the system link budget even though the wide-angle laser beacon would still be required. In practice, however, this would double or triple the system cost. The tracking system described in this paper has negligible effect on the system cost, providing at the same time times an increase in the signal by 3 to 5 . Indeed, at the given divergence, receiver aperture, and link distance we get $2.8 \mathrm{~mW}$ of intercepted laser power, which is enough for the current system. The degradation due to atmospheric distortions may be reduced by the partial compensation capability of the NLO system, although an increase of laser power is still required to overcome the losses due to beam broadening.

Increase of the communication distance may be achieved with use of NLO materials with higher nonlinearity. For example, photorefractive NLCs offer nonlinear optical coefficients up to 4 orders of magnitude larger than the thermal nonlinearity of NLCs. ${ }^{11}$ Although this increase in NLO coefficients is usually accompanied by increase in reaction time, in some cases, when high-speed tracking is not strongly required, their use may be appropriate. Use of those materials may allow the NLO lens concept to work with as little as $0.5 \mu \mathrm{W}$ of intercepted power, corresponding to an increase of link distance up to $100 \mathrm{~km}$.

\section{Conclusion}

The concept of using a dynamic nonlinear lens as an optical tracking mechanism has been suggested. Numerical modeling based on the diffraction integral showed that the narrow-angle portion of the output beam is produced by this mechanism in the range of laser power currently used in the last-mile optical links. The signal link budget improvement may be up to an order of magnitude and may allow, in particular, an increase in communication bandwidth with the same factor.

Our proof-of-concept experiments show good qualitative agreement with the concept and numerical modeling. The experiment proves that a nonlinear lens formation in a layer of NLO material in the proposed optical scheme produces a specific dynamic pattern of the optical beam passing through the material. This pattern creates a strong beam propagating in direction opposite to that of the input beam. Thus, the reversed beam tracks changes in the input beam direction. A more than fivefold signal increase at a distant receiver at $1500-\mathrm{nm}$ wavelength has been observed with a nonlinear lens formation in a nematic-liquid-crystal layer with an infrared dye. A way of improving the link efficiency and sensitivity with NLO tracking units on both communication ends is suggested. The compensation of wavefront distortions is demonstrated, showing adaptive capabilities of the device. The technique is easy to imple- 
ment and may be a cost-effective way to increase the link budget in short-distance free-space optical communication networks.

\section{Acknowledgments}

The authors thank the Space Technology Research and Development Program (STRP) of the Canadian Space Agency for financial support, and D. Gratton and Y. Arimoto for fruitful discussions. Valuable comments from two anonymous referees are greatly appreciated.

\section{References}

1. S. G. Lambert and W. L. Casey, Laser Communications in Space, Artech House, Norwood, MA (1995).

2. H. Hemmati, Ed., Deep Space Optical Communications, Wiley, Hoboken, NJ (2006).

3. J. Romba, Z. Sodnik, M. Reyes, A. Alonso, and A. Bird, "ESA's bidirectional space-to-ground laser communication experiments," in Free-Space Laser Communications IV, Proc. SPIE 5550, 287-298 (2004)

4. T. T. Nielsen and G. Oppenhaeuser, "In-orbit test result of an operational intersatellite link between ARTEMIS and SPOT4, SILEX," in Free Space Laser Communication Technologies XIV, Proc. SPIE 4635, 1-15 (2002)

5. M. Toyoshima, S. Yamakawa, T. Yamawaki, K. Arai, M. Reyes, A. Alonso, Z. Sodnik, and B. Demelenne, "Ground-to-satellite optical link tests between the Japanese laser communication terminal and the European geostationary satellite ARTEMIS," in Free Space Laser Communication Technologies XVI, Proc. SPIE 5338, 1-15 (2004).

6. A. S. Koujelev and A. E. Dudelzak, "Double phase conjugation in a liquid crystal at $1.5 \mu \mathrm{m}$ for optical beam tracking," Opt. Eng. 46(2), 024001 (2007).

7. Y. R. Shen, The Principles of Nonlinear Optics, Chap. 17, Wiley, New York (1984).

8. I. C. Khoo, Liquid Crystals: Physical Properties and Nonlinear Optical Phenomena, Wiley, New York, (1995).
9. M. Born and E. Wolf, Principles of Optics, 5th ed., Chap. 8, Pergamon Press, New York (1975).

10. B. Ya. Zel'dovich, N. F. Pilipetsky, and V. V. Shkunov, Principles of Phase Conjugation, Springer-Verlag, Berlin (1995).

11. I. C. Khoo, H. Li, and Y. Liang, "Observation of orientational photorefractive effects in nematic liquid crystals," Opt. Lett. 19, 17231725 (1994)

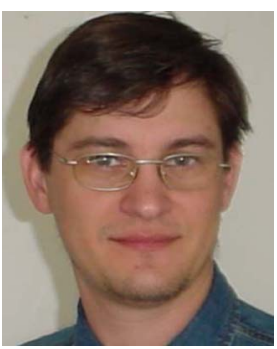

Alexander S. Koujelev received his BSc, MSc, and PhD degrees from Lobachevsky University, Nizhny Novgorod, Russia, in 1993, 1995, and 1998, respectively. In 1993, he joined the Institute of Applied Physics of the Russian Academy of Sciences as a researcher in the fields of nonlinear optics and solid-state laser techniques. From 2000, he was a visiting fellow, and since 2005 he has been a research scientist with the Canadian Space Agency, where his research activity has included nonlinear optical materials, phase conjugation, optical tracking, optical communications, laser remote sensing, and laser spectroscopy.

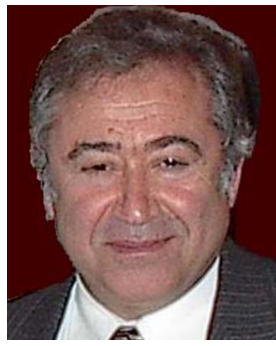

Alexander E. Dudelzak received his $\mathrm{PhD}$ degree in physics and mathematics in 1977 from the Institute of Physics, Estonian Academy of Sciences. Since 1993 he has been working at the Canadian Space Agency as a senior scientist and group leader. His areas of $R \& D$ involvement include optical spectroscopy, photonics, laser and lidar principles, and systems for real-time environmental, defense, and biomedical analytical sensing, free-space optical communications, and laser therapeutic medicine. 